International Journal of Modern Physics E

(C) World Scientific Publishing Company

\title{
TRANSPORT PROPERTIES OF A MESON GAS
}

\author{
D. FERNÁNDEZ-FRAILE* and A. GÓMEZ NICOLA ${ }^{\dagger}$ \\ Departamentos de Física Teórica I y II, Universidad Complutense, 28040 Madrid, Spain
}

Received (24 May 2007)

Accepted (12 June 2007)

\begin{abstract}
We present recent results on a systematic method to calculate transport coefficients for a meson gas (in particular, we analyze a pion gas) at low temperatures in the context of Chiral Perturbation Theory. Our method is based on the study of Feynman diagrams with a power counting which takes into account collisions in the plasma by means of a non-zero particle width. In this way, we obtain results compatible with analysis of Kinetic Theory with just the leading order diagram. We show the behavior with temperature of electrical and thermal conductivities and shear and bulk viscosities, and we discuss the fundamental role played by unitarity. We obtain that bulk viscosity is negligible against shear viscosity near the chiral phase transition. Relations between the different transport coefficients and bounds on them based on different theoretical approximations are also discussed. We also comment on some applications to heavy-ion collisions.
\end{abstract}

\section{Introduction}

Chiral Perturbation Theory (ChPT) is an effective field theory which describes the low-energy meson dynamics, based on the spontaneous breaking of the QCD chiral symmetry. The ChPT lagrangian consists of an expansion in masses and derivatives of the meson fields and perturbation theory is performed in powers of $p$ (a meson momentum, mass or temperature) over $\Lambda_{\chi} \sim 1 \mathrm{GeV}$ (for masses and momenta) or the critical temperature $T_{\mathrm{c}} \sim 200 \mathrm{MeV}$ (for temperatures). At zero temperature, the order $\mathcal{O}\left(p^{D}\right)$ of a Feynman diagram is estimated by Weinberg's power counting: $D=2+\sum_{n} N_{n}(n-2)+2 L$, where $L$ is the number of loops, and $N_{n}$ is the number of vertices coming from the lagrangian with $n$ derivatives. We will see that, for the calculation of transport coefficients, this formula does not provide the correct order for the contribution of the dominant diagrams.

In Linear Response Theory (LRT), a transport coefficient $\mathfrak{T}$ is calculated by means of Kubo-like expressions: 1

$$
\mathfrak{T}=C_{\mathfrak{T}} \lim _{q^{0} \rightarrow 0^{+}} \lim _{|\boldsymbol{q}| \rightarrow 0^{+}} \frac{\partial}{\partial q^{0}} \operatorname{Imi} \int \mathrm{d}^{4} x \mathrm{e}^{\mathrm{i} q \cdot x} \theta(t)\left\langle\left[\mathcal{A}_{\mathfrak{T}}(x), \mathcal{A}_{\mathfrak{T}}(0)\right]\right\rangle,
$$

\footnotetext{
*danfer@fis.ucm.es

${ }^{\dagger}$ gomez@is.ucm.es
} 
where $C_{\mathfrak{T}}$ is a constant, and $\mathcal{A}_{\mathfrak{T}}$ certain operator (tensorial in general). The calculation of transport coefficients is non-perturbative due to the presence in the diagrams of pair of lines with non-zero width sharing the same four-momentum. The width corresponds to the inverse of the mean collision time. These lines give rise to products of the kind $G_{\mathrm{A}} G_{\mathrm{R}} \propto 1 / \Gamma$, with $\Gamma$ the particle width. According to this, in principle, the dominant contribution to transport coefficients would come from the so called ladder and bubble diagrams. In order to take into account these non-perturbative contributions, it can be shown 1 that in ChPT is necessary to use a power counting different from Weinberg's one for diagrams with $\Gamma \neq 0$. The leading contribution $\mathfrak{T}^{(0)}$ at low temperatures corresponds to a single bubble diagram with nonzero width. For $T \gtrsim M_{\pi}$ it may be necessary to sum the ladder diagrams due to the dominance of derivative vertices. 1

In the dilute gas approximation for the pion gas, $\Gamma$ depends linearly on $\sigma_{\pi \pi}$, the pion-pion scattering cross section, that can be written in terms of partial waves $t_{I J}$ of total isospin $I$ and angular momentum $J$. A crucial point is that the behavior of transport coefficients with increasing temperature is directly linked with that of the partial waves with increasing energy and therefore the unitarity bound plays a fundamental role. ChPT violates the unitarity condition $\mathrm{SS}^{\dagger}=\hat{1} \Rightarrow \operatorname{Im} t_{I J}(s)=$ $\sigma(s)\left|t_{I J}(s)\right|^{2}$, with $\sigma(s) \equiv \sqrt{1-4 M_{\pi}^{2} / s}$ the $\pi \pi$ phase space, since partial waves contain polynomials in $s$. We will use 1 the so called Inverse Amplitude Method, which provides a exactly unitary amplitude reproducing the chiral expansion near the two-pion threshold.

\section{Electrical and thermal conductivities}

Specifically, we analyze the response from a gas against the presence of a constant electric field (DC conductivity). In this case in (1) we have $C_{\sigma} \equiv-1 / 3$ and $\mathcal{A}_{\sigma} \equiv J^{i}$ (electric current). According to Kinetic Theory (KT) $\sigma \sim e^{2} n_{\mathrm{ch}} \tau / M_{\pi}\left(n_{\mathrm{ch}}\right.$ is the density of charged particles, $\tau$ is the collision mean time, and $e$ is the particle charge), and $\tau \sim 1 / \Gamma, \Gamma \sim n v \sigma_{\pi \pi}$ ( $v$ is the mean speed of the particles). In the nonrelativistic limit, $n \sim\left(\sqrt{M_{\pi} T}\right)^{3} \mathrm{e}^{-M_{\pi} / T}, v \sim \sqrt{T / M_{\pi}}$, and $\sigma_{\pi \pi}$ is a constant, so that $\sigma \sim 1 / \sqrt{T}$. This is consistent with our result in the ChPT diagrammatic approach for $T \ll M_{\pi}$, namely $\sigma^{(0)} \simeq 15 e^{2} F_{\pi}^{4} T^{-1 / 2} M_{\pi}^{-5 / 2}\left(F_{\pi}\right.$ is the pion decay constant). The results for higher $T$ are shown in Fig. 1 (left) where we see that unitarity changes the decreasing behaviour of the DC conductivity.

The electrical conductivity is related to the soft-photon spectrum emitted by the gas of pions. Considering an hydrodynamical model of spherical symmetry in order to describe the expansion of the gas after a relativistic heavy ion collision, 1 we obtain a prediction at the origin for the photon spectrum compatible with experimental results 112

For the thermal conductivity, $C_{\kappa} \equiv-1 /(3 T)$, and $\mathcal{A}_{\kappa} \equiv T_{0 i}$. By KT: $\kappa \sim c_{p} l v$ ( $c_{p}$ is the heat capacity per unit volume and $l \sim 1 /\left(\sigma_{\pi \pi} n\right)$ is the particle mean free path). In the nonrelativistic limit, $c_{p} \sim T^{-1 / 2} \mathrm{e}^{-M_{\pi} / T}$ so that $\kappa \sim T^{-3 / 2}$. In our 

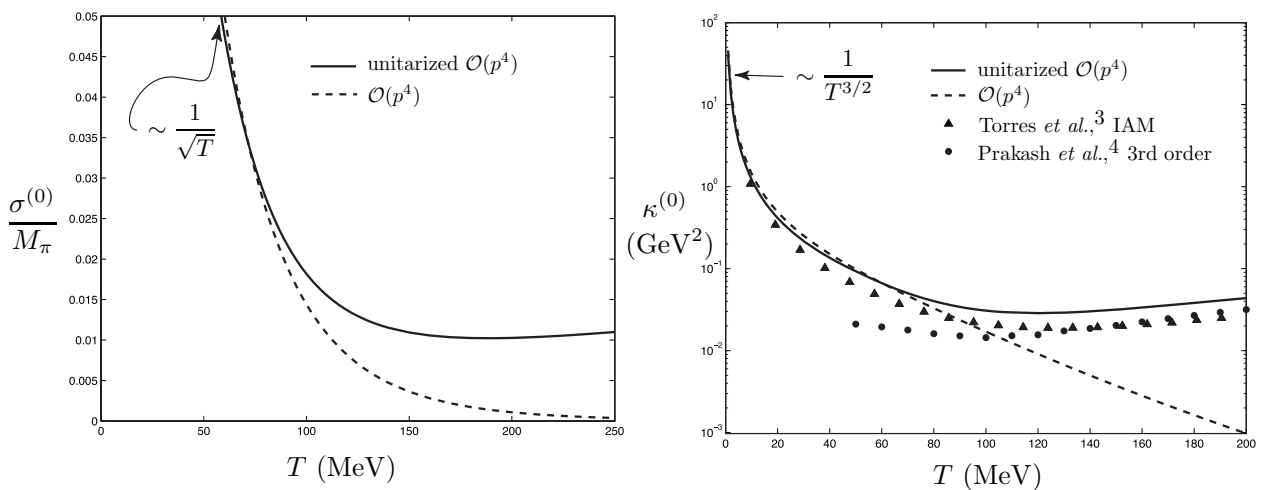

Fig. 1. (Left) Results for the DC conductivity. (Right) Results for the thermal conductivity.

ChPT scheme we obtain: for $T \ll M_{\pi}, \kappa^{(0)} \simeq 10 F_{\pi}^{4} T^{-3 / 2} M_{\pi}^{-1 / 2}$, and for $T \simeq M_{\pi}$,

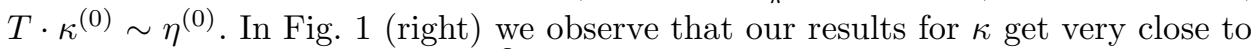
those obtained by a KT analysis 3 with unitarized amplitudes from ChPT.

\section{Shear and bulk viscosities}

The shear viscosity, $\eta$, is calculated by means of the formula (1) with $C_{\eta} \equiv 1 / 10$ and $\mathcal{A}_{\eta} \equiv T_{i j}-g_{i j} T_{l}^{l} / 3$ (non-diagonal part of the energy-momentum tensor). For the bulk viscosity, $\zeta, C_{\zeta} \equiv 1$, and $\mathcal{A}_{\zeta} \equiv-T_{l}^{l} / 3-v_{\mathrm{s}}^{2} T_{00}$, where $v_{\mathrm{s}}$ is the speed of sound in the gas. In Fig. 2 (left) we compare our results for viscosities with those obtained by Prakash et al. using a KT analysis. $\frac{4}{4}$ We also agree with a pion gas $\mathrm{KT}$ work by Dobado et al. $\frac{5}{\mathrm{~B}}$ By nonrelativistic KT we expect now the behavior $\eta, \zeta \sim M_{\pi} v n l$, so that $\eta, \zeta \sim \sqrt{T}$ and both viscosities should then be of the same order at very low temperatures. In fact, our ChPT calculation gives for $T \ll M_{\pi}$, $\eta^{(0)} \simeq 37 T^{1 / 2} F_{\pi}^{4} M_{\pi}^{-3 / 2}$, and $\zeta^{(0)} \simeq 0.36 \eta^{(0)}$ (also compatible with the analysis of Gavin 6 ). For $T \simeq M_{\pi}, \eta^{(0)} \sim 1000 \zeta^{(0)}$, so that the bulk viscosity is negligible at temperatures accessible in experiments. In fact, we get $\zeta^{(0)} \sim\left(1 / 3-v_{\mathrm{s}}^{2}\right)^{2} \eta^{(0)} \rightarrow 0$ for $T \gg M_{\pi}$, in agreement with Hosoya et al ${ }^{7}$ and Arnold et al. ${ }^{8}$

Unitarity makes the quotient $\eta / s$ ( $s$ is the entropy density) for the pion gas respect the bound $1 /(4 \pi)$ predicted by Kovtun et al., 9 as we can see in Fig. 2 (right). In addition, near $T_{c}$ our value for $\eta / s$ is not far from recent lattice and model estimates. 10 Although we do not represent it in the Figure, we do obtain a behavior for $\eta / s$ growing with $T$ for temperatures (unrealistic) $>550 \mathrm{MeV}$. A slowly increasing behavior is also obtained by calculations from the quark gluon plasma (QGP) phase 11 The sound attenuation length is given by (neglecting the contribution from the bulk viscosity) $\Gamma_{\mathrm{s}} \simeq 4 \eta /(3 s T)$, and is directly related to phenomenological effects such as elliptic flow or $H B T$ radii. We get, at $T=180 \mathrm{MeV}$, the value $\Gamma_{\mathrm{s}} \simeq 1.1 \mathrm{fm}$, in agreement with the estimate of Teaney 12 

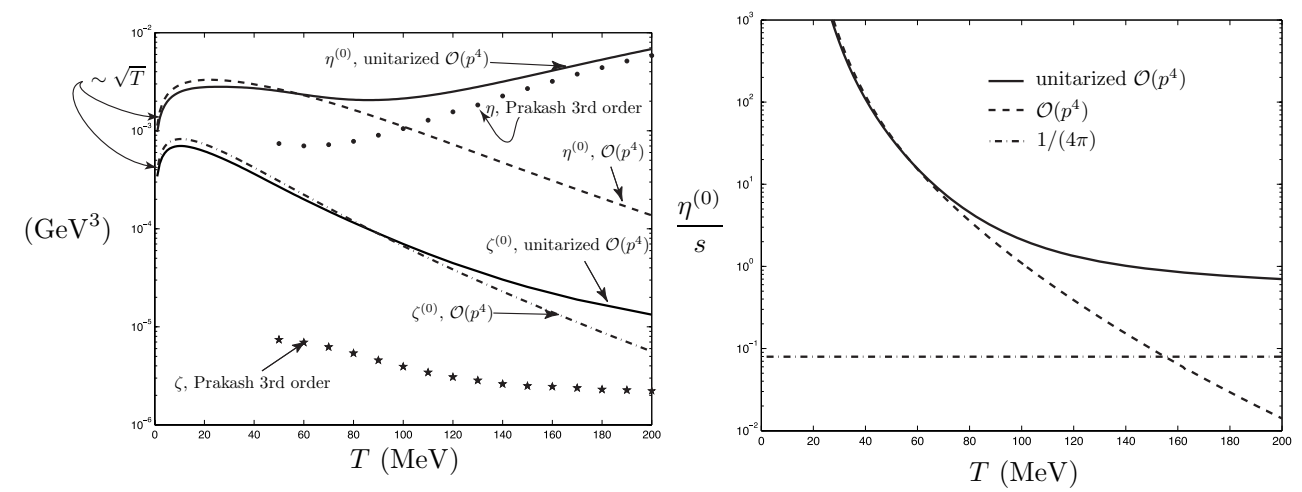

Fig. 2. (Left) Shear and bulk viscosities against temperature. (Right) $\eta / s$ quotient.

\section{Acknowledgements}

We would like to thank A. Dobado, F. J. Llanes-Estrada, and J. M. Torres Rincón for useful comments. We also thank the financial support from the Spanish research projects FPA2004-02602, PR27/05-13955-BSCH, FPA2005-02327 and the doctoral fellowship BES-2005-6726 from the MEC-FPI programme.

\section{References}

1. D. Fernández-Fraile and A. Gómez Nicola, Phys. Rev. D73 (2006) 045025; Nucl. Phys. A785 (2007) 166; Eur. Phys. J. A31 (2007) 848.

2. M. M. Aggarwal et al. (WA98 Collaboration), Phys. Rev. Lett. 93 (2004) 022301.

3. A. Dobado, F. J. Llanes-Estrada and J. M. Torres Rincón, [hep-ph/0702130].

4. M. Prakash, M. Prakash, R. Venugopalan and G. Welke, Phys. Rept. 227 (1993) 321.

5. A. Dobado and F. J. Llanes-Estrada, Phys. Rev. D69 (2004) 116004.

6. S. Gavin, Nucl. Phys. A435 (1985) 826.

7. A. Hosoya, M. Sakagami and M. Takao, Ann. Phys. 154 (1984) 229.

8. P. Arnold, C. Dogan and G. D. Moore, Phys. Rev. D74 (2006) 085021.

9. P. Kovtun, D. T. Son and A. O. Starinets, Phys. Rev. Lett. 94 (2005) 111601.

10. A. Nakamura and S. Sakai, Phys. Rev. Lett. 94 (2005) 72305; B. A. Gelman, E. V. Shuryak and I. Zahed, Phys. Rev. C74 (2006) 044908.

11. L. P. Csernai, J. I. Kapusta and L. D. McLerran, Phys. Rev. Lett. 97 (2006) 152303.

12. D. Teaney, Phys. Rev. D68 (2003) 034913. 